(Aus dem pathologischen Institut zu Freiburg.)

\title{
Studien zur Histogenese des Corpus luteum und seiner Riickbildungsproducte.
}

Von

\author{
Dr. Karl Hegar.
}

(Hierzu Tafel $X X$ und 2 Textfiguren.)

In allen Arbeiten, welche sich mit dem Corpus luteum beschäftigen, steht die Frage nach der Herkunft der Luteinzellen noch immer im Mittelpunkt der Besprechung. Während lange Zeit die bindegewebige Genese von der Mebrzahl der Autoren verfochten wurde, traten in letzter Zeit wieder Stimmen in den Vordergrund, welche, auf die an Thieren gemachten Untersuchungen von Sobotta u. A. sich stützend, die vorwiegend epitheliale Abstammung auch beim Menschen verfochten. Doch stossen alle diese Versuche anf die Schwierigkeit, ja Unmöglichkeit, fortlaufende Stadien der gelben Körper bei dem Menschen zu erhalten.

In jüngster Zeit hat $\mathrm{Cohn}^{1}$ ) den Versuch gemacht, den Nachweis der Abstammung der typischen Luteinzellen aus dem Granulosaepithel zu führen. Es ist sein Verdienst, an einem grossen Material beim Menschen genauer das regelmässige Vorkommen von zwei verschiedenen Formen von Luteinzellen festgestellt zu haben, die zuerst genauer von Rabl beschrieben sind, nämlich eine innere, die Hauptmasse der charakteristischen grossen Luteinzellen umfassende Schicht, die er von den Granulosazellen ableitet, und eine äussere, weniger gleichmässig entwickelte, in ihren Zellformen sich deutlich von der ersteren unterscheidende, die sogen. Thecaluteinschicht, die, von der Theca interna abstammend, als Matrix der bindegewebigen Organisation dienen soll.

1) Cohn, Ueber das Corpus luteum. Dieses Arch. Bd. 87. 
Ich habe nun das mir zur Verfügung stehende Material einer Durchsicht unterzogen mit besonderer Berücksichtignng der von Cohn und seinen Vorgängern berührten Verhältnisse und dabei nur gut erhaltene operativ gewonnene Präparate, die keine erkennbaren pathologischen Verhältnisse darboten, ausgewählt. Ein Theil der Präparate, darunter diejenigen, die ich zur Beurtheilung der Rückbildungsvorgänge der Follikel abbilde, entstammen der Sammlung des Herrn Prof. Aschoff, dem ich für die Ueberlassung derselben meinen verbindlichsten Dank ausspreche.

Ich konnte dabei die Befunde Cohn's zum grossen Theil bestätigen, einzelne Bilder jedoch in meinen Präparaten bieten ziemliche Abweichungen dar, oder lassen auch andere Deutungen zu; vor allem aber kann ich den zu weit gehenden Sehlüssen, welche Cohn in seiner Arbeit ziebt, nicht folgen.

Von nicht geringer Bedeutung scheint mir ein Präparat zu sein, dessen Beschreibung ich hier voranstellen möchte:

1. Frau J. Operation 2 Tage vor dem erwarteten, ein Tag vor dem wirklichen Eintritt der Periode. Retroflexio fixata, nur leichte Verwachsungen der rechten Adnexe, die entfernt werden. Das linke Ovarium enthielt keinen sichtbaren Follikel. Das rechte enthält ein altes, schon weit bindegewebig umgewandeltes Corpus luteum, das wohl der vorhergehenden Menstruation zuzuschreiben ist und einen ganz frischen, anscheinend unmittelbar vor der Operation geplatzten Follikel. Die Rupturstelle ist makroskopisch wie mikroskopisch gut zu erkennen. Das ganze Gebilde ist $1 \mathrm{~cm}$ lang und $1 / 2 \mathrm{~cm}$ breit, die Längsaxe verläuft der Oberfläche des Eierstocks parallel. Die Einrissstelle wird dargestellt durch einen $1 \mathrm{~mm}$ langen feinen Canal, beinahe in der Mitte der Länge des Follikels. Die Wände des Canals werden von stark blutig durchtränktem Bindegewebe gebildet, an seinem Ausgang haftet locker eine kleine Schicht losgerissenen Epithels. An der inneren Mündung des Canals finden sich in der Wandbekleidung erst vereinzelte Luteinzellen, welche dann in eine zusammenhängende Lage übergehen. Etwas weiter entfernt von der inneren Oeffnung beginnt auch wieder eine intacte Epithelbekleidung der Innenfläche, die sich im ganzen Umfang des Follikels mehr oder weniger dicht erhalten hat. An einzelnen Stellen ist sie wohl mechanisch von der Unterlage abgelöst und schwimmt frei in der Höhlung; ein Ei wurde in keinem Schnitte gefunden. Die Luteinschicht ist ebenfalls im ganzen Umfang des gelben Körpers in verschiedener Mächtigkeit erhalten, an manchen Stellen stark von Hämorrhagien zerrissen, an anderen in mehrfacher Lage gut erhalten. Sie ist überall von dem Epithel, soweit es nicht traumatisch abgelöst erscheint, durch eine scharfe Membran mit blutgefüllten Gefässen getrennt. Eine Durchbrechung dieser Membran durch Luteinzellen oder durch einwuchernde Gefässe ist nirgends festzustellen. Die Luteinschicht besteht aus den typischen, grossen, polygonalen Zellen mit grossem Protoplasmahofe, die freilich etwas kleiner, als im fertigen 
gelben Körper sind. Sie bilden einen einheitlichen Wall um das Follikelepithel.

Nach aussen von dieser Luteinzellschicht, jedoch nicht im ganzen Umfang des Körpers, finden sich Zellformen, die deutlich von den ersteren sich unterscheiden, sie sind kleiner, liegen enger aneinander und haben dunkleren Protoplasmahof. Die Anordnung ist eine ungleichmässige, fleckweise, zeigt aber deutlich eine gewisse Beziehung zu den Gefässen. Die Zellhaufen finden sich mit Vorliebe da, wo Gefässe in die Luteinschicht eintreten. Die äussere Abgrenzung gegen die Theca externa ist durch die Durchblutung des Gewebes eine scharfe (siehe Taf. XX, Fig. 1).

Es scheint mir keinem Zweifel zu unterliegen, dass diese perivasculären Zellhaufen den Anfangsstadien der von Cobn beschriebenen äusseren Zellschicht entsprechen.

Wir haben also hier, was besonders zu betonen ist, in einem noch mit Epithel ausgekleideten Follikel bereits eine deutliche Doppelschichtung der Luteinzellen, eine innere, völlig zusammenhängende und eine äussere, mehr fleckförmig angeordnete Lage.

Die folgenden Präparate betreffen Follikel, bei denen keine Epithelschicht mehr zu finden ist. Die Entwickelung der Luteinschicht hat bereits zu makroskopisch erkennbaren Corpora lutea geführt, bei denen freilich der gelbe Saum sehr verschieden starke Dickendurchmesser aufweist.

Alle diese Eierstöcke wurden wenige Tage vor oder nach der Menstruation entfernt. Leider ist ja bis heute eine genaue Altersbestimmung der gelben Körper nicht möglich. Wir wissen über den Zeitpunkt der Follikelruptur noch immer nichts Sicheres, und wenigstens nach meinen Beobachtungen kann die Luteinzellschicht eines ganz frischen Corpus luteum dicht vor der Menstruation schon die gleiche Stärke der Entwickelung aufweisen, wie direct nach der Periode.

Dazu kommt noch, wie ich im Gegensatz zu anderen Auffassungen betonen muss, dass die Rückbildungsperioden der Corpora lutea sich über mehrere Menstruationsperioden erstrecken, die Rückbildung auch anscheinend verschieden schnell sich abspielt, so dass die Altersbestimmung sehr erschwert wird.

Bei den jetzt zu beschreibenden Präparaten handelt es sich stets um Follikel, die zwar nicht ganz frisch rupturirt waren, da das Epithel bereits fehlte, die sich aber noch in den ersten Stadien der Umwandlung zum gelben Körper befanden, weil die Luteinzellschicht, wenn auch schon makroskopisch erkennbar, doch noch 
relativ schmal, und wie die zu beschreibenden Bilder lehren, in der Fortentwickelung, nicht in der Rückbildung begriffen war.

2. Ga. Operation unmittelbar vor Eintritt der erwarteten Periode. Der Follikel ist anscheinend noch nicht lange geplatzt und bildet einen $2 \mathrm{~cm}$ langen, in seiner centralen Partie zusammengefallenen, im peripheren Abschnitt eine kreisrunde, $1 \mathrm{~cm}$ im Durchmesser haltende Höhle dar. stellenden Körper. Von der Eierstocksoberfläche ist diese Höhle durch eine $1 \mathrm{~mm}$ breite, wie eine frische Narbe aussehende, bindegewebige Brücke getrennt. Der Inhalt ist frisches Blut und Fibrin. Die Luteinschicht kleidet nur den zusammengefallenen Theil des Körpers und etwa das innere Drittel der erweiterten Höhle aus. Sie ist deutlich entwickelt, 1-2 mm dick.

Auch hier fällt eine Zweitheilung ganz wie bei dem frischgeplatzten Follikel sofort in die Augen. Die äussere Zellschicht ist uur in den Faltungswinkeln des Luteinzellmantels, da wo die Gefässe in ihn eintreten, deutlich erkennbar. Bei starker Vergrösserung verschwindet der Unterschied beinabe vollkommen. Feine neugebildete Bindegewebssprossen durchziehen die Luteinschicht und bilden an ihrer Innenfläche eine zusammenhängende Hembran.

3. Schw. Operation 3-4 Tage vor der erwarteten Periode. Das Corpus luteum bildet einen kirschgrossen, $1 \mathrm{~cm}$ langen, $3 / 4 \mathrm{~cm}$ breiten Körper, mit nur leicht gefalteter Wand. Auch hier ist die Zweitheilung der Zellschicht bereits ausgeprägt. Die äussere ist nur fleckweise und in den Faltungswinkeln, da aber deutlich entwickelt und setzt sich ziemlich scharf von den eigentlichen Luteinzellen ab. Im Inneren frisches Blut und beginnende Bindegewebsentwicklung.

4. Wi. Das Corpus bildet eine im Durchschnitt halbmondförmige, $11 / 2 \mathrm{~cm}$ lange, $3 / 4 \mathrm{~cm}$ breite Höhle, die von einer schmalen Luteinmembran gleichmässig ausgekleidet ist. Die zwei Schichten lassen sich beinahe im ganzen Umfang deutlich unterscheiden; die äussere Schicht erscheint auch hier am deutlichsten in der Limgebung der Gefässe. Die Zellen unterscheiden sich von denen der inneren Schicht lediglich durch kleineres Kaliber und geringere Färbung des Protoplasma, bei starker Vergrösserung verschwindet der Unterschied nahezu vollkommen. Der Inhalt ist Serum, eine Bindegewebsentwicklung fehlt noch vollkommen.

5. Hy. Operation am 1. Tag der Periode. Grösse des gelben Körpers $1 / 2-1 \mathrm{~cm}$. Luteinschicht mit beiden Zellformen schon deutlich entwickelt, die äussere Schicht am deutlichsten in den Faltungswinkeln. An den Septen der Rindenschicht ist der Uebergang der beiden Zellformen ineinander ein ganz allmählicher. Nach iunen ist die Luteinschicht von einer feinen Membran jungen Bindegewebes mit frischem Blut ausgekleidet.

6. Ba. Operation am 2. Tag der Periode. Der Befund ist ziemlich der gleiche, wie in dem vorhergehenden Präparate. Nur ist das Bild durch starke Füllung der Gefässe und freie Blutergüsse ins Gewebe etwas verwischt, $d a$ bei der vaginal vorgenommenen Operation der Eierstock sich nicht ohue stärkeren Druck entfernen liess. Die Bindegewebsneubildung innerhalb der Luteinschicht und an ibrer inneren Begrenzung ist schon reichlicher, wie in dem vorigen Präparate.

In einer weiteren Reihe von untersuchten Präparaten handelt es sich um schon weiter vorgeschrittene Stadien des gelben Körpers. 

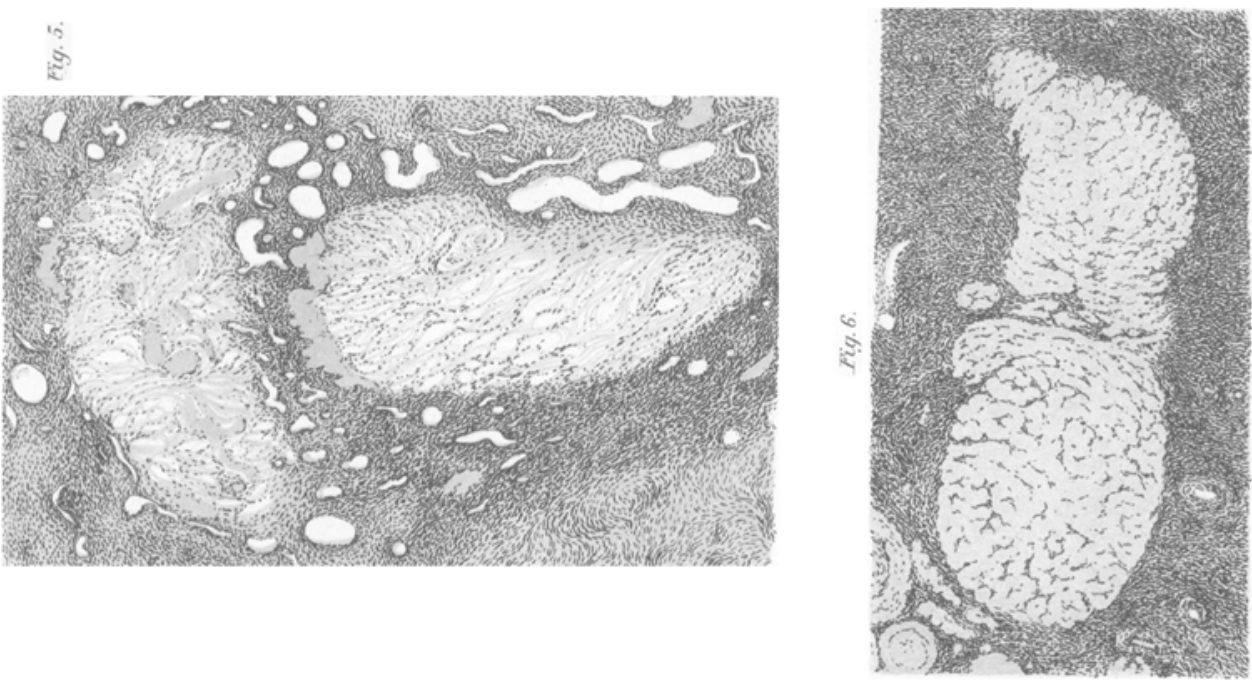

\section{క}

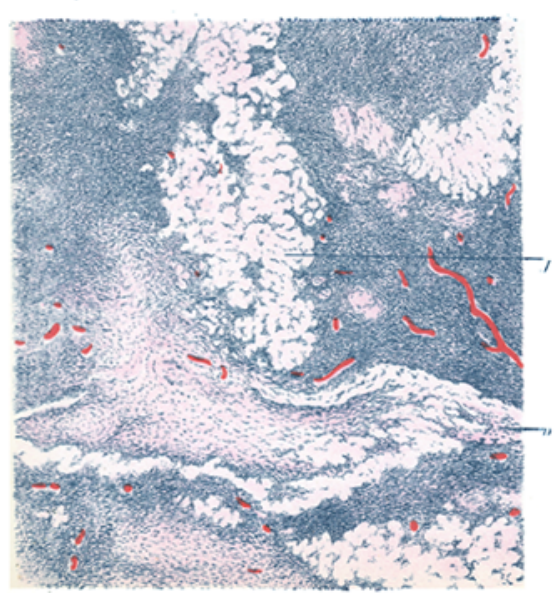

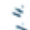

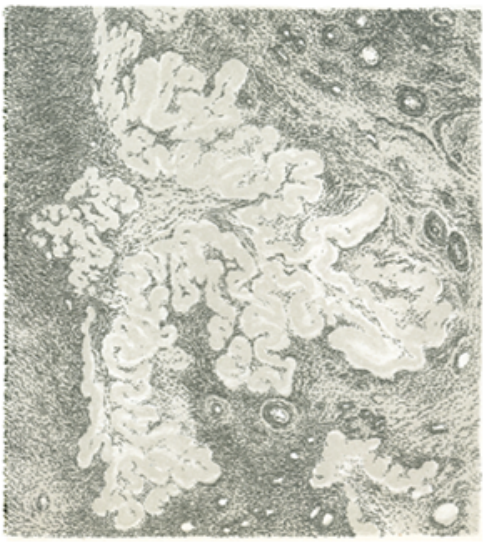

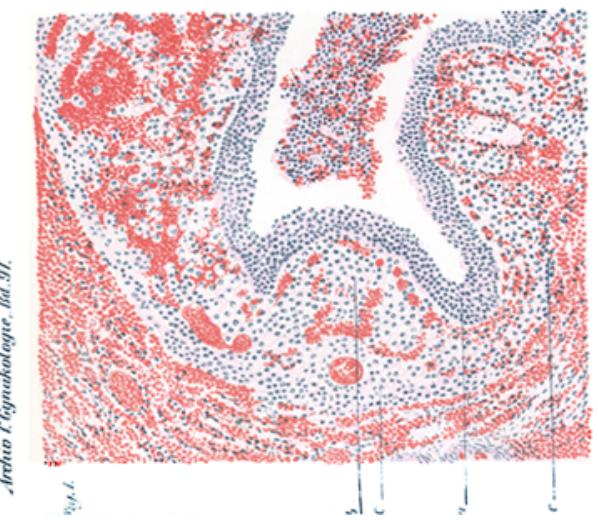

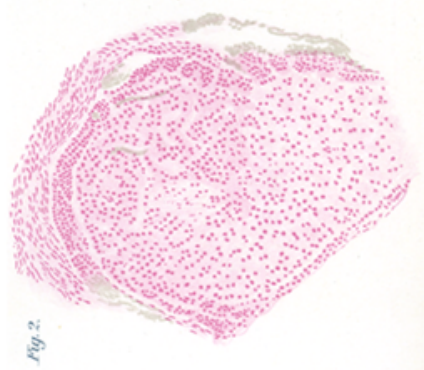


In allen ist die äussere Luteinschicht mehr oder weniger stark und deutlich entwickelt, nie im ganzen Umkreis des Follikels, meist fleckweise und besonders in der Umgebung der Gefässe. Es besteht schon eine reichliche Entwickelung jungen Bindegewebes innerhalb der Luteinschicht und in ihrer Begrenzung nach der Höhlung. Diese ist im Centrum meist leer, am Rande findet sich stets frisches Blut.

Es nimmt also unter den oben geschilderten Präparaten das erstgenannte eine Sonderstellung ein, $d a$ es sich anscheinend um ein sehr frühes Stadium der Entwicklung des gelben Körpers handelt. Leider lassen sich die Einwirkungen des Operationstraumas hier nicht ganz ausschliessen, sie seheinen mir aber nicht so bedeutend, dass der Charakter des histologischen Bildes dadurch wesentlich beeinflusst, oder unkenntlich gemacht worden wäre.

Bei unserem Präparate ist die Epithelschicht noch nahezu im ganzen Umkreis erhalten, oder nur mechanisch von der Unterlage getrennt, der Follikel scheint gerade kurz vor der Operation geplatzt oder vielleicht durch die Operation za etwas vorzeitigem Bersten gebracht worden zu sein. Das Epithel zeigt keine Spuren von Degeneration, aber auch keine Anzeichen einer Umwandlung in Luteinzellen, es ist überall, wo nicht eine traumatische $\mathrm{Ab}$ lösung stattgefunden hat, noch durch eine scharfe Grenze von der unterliegenden Schicht getrennt, die zahlreiche feine Gefässe enthält. Weiter finden wir in der Luteinschicht deutlich beide von Rabl, Cohn u. a. beschriebenen Zellformen vertreten, nicht in gleicher Stärke und Vertheilung, aber immerhin in ähnlicher Anordnung, wie in älteren gelben Körpern.

In allen anderen Präparaten finden wir, übereinstimmend mit Cohn die beiden Zellarten vertreten, von denen die eine als Granulosa-, die andere als Thecaluteinschicht von diesem Autor aufgefasst wird, welch letztere mit dem Alter des Gebildes an Menge und Deutlichkeit abnimmt.

Bei Besprechung der Frage nach ihrer Genese scheint mir vor allem das Verhalten der Grenzen zwischen den Zellschichten und dem Abschluss nach der Höhle, und weiterhin die Mengenvertheilung der äusseren Schicht von Wichtigkeit.

Das Granulosaepithel grenzt sich beim reifen Follikel durch eine deutliche, nach Hörmann aus feinen Fibrillen bestehende, Basalmembran scharf von der Theca interna ab. In unserem frühen Stadium ist diese Membran an allen Stellen, wo das Follikel- 
epithel nicht abgelöst ist, noch unverändert vorhanden, da wo das Epithel mechanisch weggeschwemmt ist, wird der Hohlraum von der Membran begrenzt. Was aus dieser Basalmembran wird, entzieht sich bis jetzt der Beobachtung. Bei einer Umwandlung des Epithels in Luteingewebe müssten wir doch auch im fertigen Corpus luteum noch eine Grenzschicht gegen die Thecaluteinzellen feststellen können, das ist aber nicht der Fall. So deutlich sich auch oft die sogenannten Granulosa- von den Thecaluteinzellen unterscheiden, so sind sie doch nie durch eine besondere Grenzschicht getrennt. Sie liegen wohl nebeneinander, bilden aber zusammen ein einheitliches Gewebe. Auch treten ja mit zunehmendem Alter des Körpers die Thecazellen mehr und mehr an Menge zurück, um schliesslich ganz zu verschwinden, was wahrscheinlich auf allmählicher Umwandlung der Theca- in Granulosazellen beruht.

Umgekehrt finden wir in allen gelben Körpern, soweit sie bereits der Epithelauskleidung verlustig gegangen sind, selbst in den frühesten Stadien die innere Luteinzellschicht gegen die Höhlung des Corpus luteum von einer feinen bindegewebigen Membran begrenzt, welche unzweifelhaft der ursprünglichen Grenzmembran zwischen Theca interna und Granulosaepithel entspricht. Würden die Granulosaluteinzellen aus dem Granulosaepithel hervorgehen, so müsste wenigstens vorübergehend ein Entwicklungsstadium gefunden werden, wo diese angebliche epitheliale Schicht noch frei gegen das Lumen angrenzt. Unter meinem Materiale habe ich nur einmal einen Fall gesehen, in welchem die bindegewebige Grenze so undeutlich hervortrat, dass man, aber auch nicht im ganzen Umkreis des Hohlraumes, von einer freien Oberfläche der Granulosaluteinzellenschicht hätte sprechen können. Da aber dieser Fall nicht einwandsfrei das Fehlen der Grenzmembran beweist und ich bisher keinen zweiten Fall gefunden habe, so scheint mir vorläufig die freie Begrenzung der Granulosazellen nicht bewiesen, auch in den Beschreibungen und Abbildungen der früheren Autoren nirgends mit Sicherheit begründet.

Während die Existenz einer solchen Grenzmembran es doch recht zweifelhaft erscheinen lässt, ob die epitheliale Genese der Granulosaluteinzellen wirklich so gesichert ist, wie man es heute annimmt, ob es wirklich erlaubt ist, die exakten und unzweifelhaft sehr wichtigen Ergebnisse der Sobotta'schen Untersuchungen auf den Mensehen zu übertragen, so deuten umgekehrt die Ver- 
hältnisse zwischen den beiden Luteinzellschichten darauf hin, dass sie gemeinsamer, d. h. bindegewebiger Abkunft sind.

Die Ausbreitung der Thecaluteinschicht ist in den fertigen gelben Körpern eine sehr ungleiche, jedenfalls bildet sie nie einen geschlossenen Ring, sondern tritt mehr fleckenförmig auf, besonders im Anschluss an die Gefässe, während die Luteinschicht in dem Präparat mit erhaltenem Epithel bereits einen geschlossenen Ring typischer Luteinzellen bildet.

Wir haben auch gesehen, dass der Typus des Thecaluteingewebes durchaus nicht immer so einheitlich und so scharf von der inneren Luteinschicht zu unterseheiden ist. Es lassen sich sehr wohl verschiedene Zellformen und auch Uebergänge zwisehen beiden Arten erkenven; in Taf. XX, Fig. 2 bilde ich eine Stelle aus einem Corpus luteum graviditatis ab, die ich der Arbeit Sohma's ${ }^{1}$ ) entnehme. Hier finden sich deutlich, zum Theil in allmählichem Uebergange sogar drei verschiedene Zellarten, die sehr wohl einheitlichen Ursprungs sein können.

Besonders auffallend ist auch das, von Cohn ebenfalls erwähnte, Verschwinden des Untersehieds der Zellen bei der Betrachtung mit starker Vergrösserung. Man kann dann sehr oft in Präparaten, in denen mit schwacher Vergrösserung noch eine haarscharfe Grenze zwischen den beiden Schichten zu bestehen scheint, diese nicht mehr erkennen, und es verschwindet vor allem jeder qualitative Onterschied zwischen den Zellen.

Auch Seitz ${ }^{2}$ ) hat auf dieses Verhalten aufmerksam gemacht und eine solche Stelle aus einem sehr jungen gelben Körper abgebildet. Er schreibt dazu: „An der einen Stelle glaubt man den sicheren Uebergang der epitheloiden Zellen in Luteinzellen feststellen zu können, an der anderen spricht alles dafür, dass die Luteinzellen aus epithelialen Elementen hervorgegangen sind. Bei der richtigen Auswahl wird jeder je nach seinem Standpunkt im Stande sein, eine Stelle ausfindig zu machen, die für sich allein berücksichtigt, die bindegewebige oder die epitheliale Genese der Luteinzellen zu beweisen scheint."

Gerade der Befund dieser versehiedenen ineinander übergehenden Zellformen, ferner die ungleichmässige Vertheilung der äusseren

1) Sohma, Dieses Archiv. Bd. 84 .

2) Seitz, Die Follikelatresie während der Schwangerschaft. Dieses Archiv. Bd. 77 . 
Zellschicht mit Anhäufung in den Septen und um die Gefässe, ihr Versehwinden in älteren Stadien, lassen die Vermuthung nicht unterdrücken, dass die Zellarten vielleicht doch einheitlicher Genese sind, und ihre verschiedenen Formen nur aufeinander folgende Entwicklungsstadien sind und dass die Unterschiede vielleicht nur durch mechanische Verhältnisse bedingt sind, da ja bei dem Zusammenfallen des Follikels, der wechselnden Menge des Inhalts, späteren Nachblutungen Druckwirkungen verschiedenster Art auf die Zellen ausgeübt werden.

Zusammenfassend können wir über diese Verhältnisse aus unseren Präparaten Folgendes ersehen:

Es lässt sich bei noch erhaltenem Epithel eines geplatzten Follikels schon eine deutliche Luteinzellenschicht feststellen mit den grossen, typischen Luteinzellen und an deren äusseren Peripherie kleineren den sogenannten Thecaluteinzellen ähnlichen Zellformen. Bei älteren gelben Körpern lassen sich die von Cohn u. a. beschriebenen zwei Arten von Luteinzellen stets erkennen, doch ist die äussere Schicht nie in geschlossenem Verbande, sondern nur fleckförmig im Anschluss an die Gefässe vorhanden, und es lassen sich allmähliche Uebergänge der beiden Zellarten ineinander nicht verkennen.

Ich möchte damit keineswegs eine feste Entscheidung über die Genese der Luteinzellen treffen. Diese kann erst nach Auffindung einer grösseren Reihe von Uebergangsstadien gefällt werden. Vor allem ist die Frage nach dem Schicksal des Granulosaepithels noch zu lösen, ob es ebenfalls zu Grunde geht oder sich an der Bildung des Luteingewebes betheiligt.

Jedenfalls aber muss ich mich gegen die Schlüsse von Cohn wenden, welcher diese so schwierige Frage der Abstammung der Luteinzellen einfach auf Grund des Vorkommens einer doppelten Luteinzellenschicht auch im Sinne der doppelten Abkunft lösen zu dürfen glaubt. Wie ich gezeigt habe, kann diese doppelte Zellschichtbildung mit viel grösserer Wahrscheinlichkeit auf eine einheitliche und zwar wahrscheinlich bindegewebige Abkunft zurückgeführt werden.

Die Forscher, die sich bisher mit dieser Frage beschäftigt haben, drücken sich über die Genese im Ganzen recht zurückhaltend aus, so hat z. B. Rabl den Schluss auf die doppelte Genese nicht gezogen, sondern vielmehr ebenfalls betont, dass der 
Unterschied bei starker Vergrösserung weniger deutlich wird und ein allmählicher Uebergang nicht unwahrscheinlich ist.

Van der Stricht geht etwas weiter und lässt wenigstens die Wahrscheinlichkeit einer doppelten Genese vermuthen. Von neveren Kritikern nähert sich Schottländer ${ }^{1}$ ), der bisher für den rein bindegewebigen Ursprung eingetreten war, der dualistischen Auffassung, thält aber die reinliche Scheidung zwischen beiden Zellarten vorläufig nicht für durchführbar. Bayer ${ }^{2}$ ) spricht sich aus dem Grunde für die grössere Wahrscheinlichkeit der epithelialen Genese aus, da mit ihr die moderne Auffassung des gelben Körpers als Drüse mit innerer Secretion stehe und falle.

Cohn hat nun den Versuch gemacht, auch histologisch die Lehre von der epithelialen Abstammung zu stützen. Aber auch in seinem grossen Material finden sich ausschliesslich fertige gelbe Körper, ein entscheidendes Uebergangsstadium hat auch er nicht beobachtet. Seine sehr ausführliche Beweisführung gipfelt in dem Satze $^{3}$ ): „Es gleichen auch im menschlichen gelben Körper die grossen, unregelmässig polygonalen Zellen vollkommen den Luteinzellen, deren continuirliche Entwickelung aus den Granulosaepithelien bei einer Anzahl von Thierarten an fortlaufenden Entwicklungsstadien einwandsfrei beobachtet worden ist." So wünschenswerth es wäre, beim Menschen die analoge Entwicklung wie bei Thieren annehmen zu dürfen, so kann diese indirecte Beweisführung uns doch nicht genügen, und wir müssen die Frage noch offen lassen, bis eine grössere Anzahl einwandsfreier Uebergangsstadien bekannt sein werden.

Das Verhältniss von 0 vulation zu Menstruation gestaltet sich in meinen Beobachtungen folgendermaassen:

In 4 Fällen wurde die Operation kurz vor der erwarteten Periode ausgeführt, jedesmal war das Corpus luteum noch frisch, so dass die Ovulation unmittelbar vorber oder nur ganz kurze Zeit vorher erfolgt sein konnte. Am 1. und 2. Tag der Menstruation war zweimal operirt worden, beidemal handelte es sich um etwas ältere gelbe Körper. Bei den übrigen im Intermenstruum operirten Fällen entsprach das Corpus etwa dem Zeitpunkt der letzten Menstruation. Jedenfalls fand sich ein frischgeplatzter Follikel

1) Schottländer, Referat über Pannenstiel, Die Erkrankungen des Eierstockes. Monatsschr. f. Geb. Bd. 30.

2) Bayer, Vorlesungen über allgemeine Geburtsbülfe. Strassburg.

3) 1. c. S. 396 . 
nur vor der Menstruation, bei gleichzeitiger Anwesenheit auch älterer Stadien. Mit Ausnahme von zwei Fällen lagen also Ovulation und Menstruation zeitlich nahe zusammen, meist ging die Follikelberstung dem Eintritt der Periode unmittelbar voran. Gerade in den Beobachtungen, in denen die Operation während der Regel gemacht wurde, waren die gelben Körper älteren Datums, wenigstens fanden sich auch in dem anderen Eierstock keine jüngeren Stadien.

Die Bestimmung des Alters eines Corpus luteum nach seiner histologischen Beschaffenheit lässt sich naturgemäss, wie wir schon oben bemerkten, nie mit voller Sicherheit ausführen. Als Anhaltspunkte dienen die Entwickelung der Luteinschicht, die mehr oder weniger vorgeschrittene bindegewebige oder hyaline Umwandlung; von den Anfangsstadien können wir nur vermuthen, dass die Luteinschicht sich sehr schnell, wahrscheinlich in den ersten Stunden nach der Entleerung, entwickelt.

Leopold und Ravano ${ }^{1}$ ) haben nun als Maass der Altersbestimmung lediglich das Verhalten des primären Blutergusses und seine Ersetzung durch die sich bildende Luteinschicht und weiterhin durch Bindegewebe benützt und so ganz schematisch das Alter der von ihnen zur Klarlegung des Verhältnisses von Ovulation zur Menstruation verwertheten Corpora lutea bestimmt. Darnach entsteht die Rinde des gelben Körpers erst im Laufe der zweiten Woche. Wenn die Luteinschicht thatsächlich so lange zu ihrer Entwickelung brauchte, so wäre es nicht schwer, die Uebergangsstadien aufzufinden, und die Frage nach der Genese der Luteinzellen wäre längst gelöst. Leider wird aber in den Fällen der genannten Autoren, bei denen es sich um Corpora lutea der ersten Wochen handeln soll, nur bemerkt, dass der Inhalt bloss aus Schleim und Blut bestehe, eine Rinde aber noch nicht vorhanden sei, über das Schicksal des Epithels und das histologische Verhalten der Wandbekleidung erfahren wir nichts. Es erscheint fraglich, ob es sich bei einigen dieser als Frühstadien bezeichneten Corpora haemorrhagica nicht um pathologische Bildungen gehandelt hat.

Ich möchte auf die Berechtigung der von Leopold und

1) Leopold und Ravano, Neuer Beitrag zur Lehre von der Menstruation und Ovulation. Dieses Mrchiv. Bd. 83. 
540 Hegar, Studien zur Histogenese des Corpus luteum u.s.w.

Ravano geübten Altersbestimmung hier nicht näher eingehen und nur der Frage des Blutergusses einige Bemerkungen widmen.

In den von mir beobachteten, noch nicht lange geplatzten Follikeln findet sich mit einer Ausnahme relativ wenig oder fast gar kein Blut, in dem frühesten Stadium ist hauptsächlich die Wand durchblutet, was sich zum Theil wohl auch durch das Trauma erklären lässt. Dagegen ist in den nach ihrer histologischen Beschaffenheit schon älteren Follikeln mehr Blut und $z$ war frisehes besonders im Bereich der die Innenwand auskleidenden jungen Bindegewebsschichte zu finden. Dies stimmt überein mit der Beobachtung Cohns, der die Blutansammlung häufig nicht im Centrum, sondern nur am Rande fand, während die Nitte leer oder mit blutfreier Flüssigkeit erfüllt war.

Wenn also auch das Corpus luteum zu Hämorrhagien in hohem Maasse disponirt ist, so scheint es doch nicht die Regel zu sein, dass stets eine sehr heftige Blutung in den Inhalt des Follikels erfolgt. Auch noch ganz junge gelbe Körper, wie der in No. 4 beschriebene können ganz oder nahezu frei von Blut sein.

Da nun die Periode in den meisten Fällen der Ovulation erst in mehr oder weniger kurzer Zeit nachzufolgen pflegt, so scheint mir die Vermuthung nicht ganz unberechtigt zu sein, dass die Blutung erst secundär in den Hohlraum oder in die Wandschichten erfolgt. Die ersteintretende und eventuell die nächstfolgende Menstruation würden dann dazu dienen, die Involution des gelben Körpers zu beschleunigen. Damit würde übereinstimmen, dass beim Corpus luteum graviditatis die Involution sehr verlangsamt ist, da in der Schwangerschaft die menstruelle Congestion ausbleibt und die Follikelreifung nur unvollkommen erfolgt.

Bei der Schilderung der Rückbildungsvorgänge und Producte der Eierstockfollikel ist man bestrebt gewesen, scharf zu unterscheiden zwisehen denen der Corpora lutea und denen des atretischen Follikels. Dennoch liegt es auf der Hand, dass eine genaue Trennung beider nie vollständig durchzuführen sein wird, da je nach der Grösse und dem Entwicklungsgrade des ursprünglichen Gebildes auch die Rückbildung sich verschieden gestalten muss.

Ich möchte mich in dieser Hinsicht den Ausfübrungen Böshagens ${ }^{1}$ ) anschliessen, dessen Eintheilung wohl allen vorkommen-

1) Böshagen, Ueber die verschiedenen Formen der Rückbildungsproducte der Eierstockfollikel u. s. w. Zeitschr. f. Geb. Bd. 53. 
den Fällen gerecht wird und genügenden Spielraum für die unvermeidlichen Variationen lässt. Nach ihm sind es drei Momente, die wesentlich die Art der Rückbildung beeinflussen, nämlich die Grösse des ursprünglichen Gebildes, sei es nun ein Corpus luteum oder ein atresirender Follikel, ferner die Menge des in den Hohlraum einwuchernden oder in ihm sich entwickelnden Bindegewebes, und endlich der Grad der Ausbildung und der hyalinen Degeneration der Theca interna.

Cohn wirft Böshagen. vor, dass er bei seiner Eintheilung den nicht anzuerkennenden Untersehied zwischen Corpus luteum graviditatis und menstruationis wieder einführe. Dass dieser Unterschied qualitativ nicht besteht, ist allgemein anerkannt, aber das Corpus luteum graviditatis pflegt in seinen Dimensionen und seiner Lebensdauer das der Menstruation sehr zu übertreffen. Die Grösse des ursprünglichen Gebildes spielt aber bei der Gestaltung des Rückbildungsvorganges eine sehr wichtige Rolle, und so hat die obige Unterscheidung doch eine gewisse Berechtigung.

Umgekehrt werden bei der Rückbildung der Corpora lutea (menstruationis) und der atretischen Follikel die Grenzen wieder sehr verwischt, da bei beiden der Grad der Ausbildung und späteren hyalinen Umwandlung der Theca interna entscheidend für die Form des Rückbildungsproductes ist.

Da bei beiden die Theca interna sich sehr umfangreich entwickeln und mehr oder weniger stark hyalin entarten kann, so können die gleichen oder wenigstens sehr ähnliche Rückbildungsproducte sowohl aus geplatzten, wie aus atretischen Follikeln entstehen, und es treten auch nicht selten Mischformen auf, bei denen die Herkunft besonders in vorgerückteren Stadien nicht mehr mit Sicherheit zu bestimmen ist. So lässt sich die scharfe Trennung, wie Seitz und Cohn sie vorschlagen, nicht durchführen.

Es kann also aus einem gewöhnlichen atresirenden Follikel das einfache Corpus atreticum mit oder ohne hyalines Band hervorgehen, je nachdem eine grössere Thecaluteinschicht bestanden hat oder nicht. Fig. 1 zeigt nebeneinander zwei (nicht zusammengehörige) solehe Rückbildungsproducte, das eine ist lediglich eine durch etwas lockeres Bindegewebe angedeutete Narbe, von dem anderen ist nur ein unregelmässig zackiges, hyalines Band übriggeblieben. In Fig. 2 ist ein atretischer Follikel aus dem Ovarium eines Neugeborenen abgebildet, ein Corpus fibrosum mit schmalem hyalinen Bande. 
542 Hegar, Studien zur Histogenese des Corpus luteum u.s.w.

Figur 1.

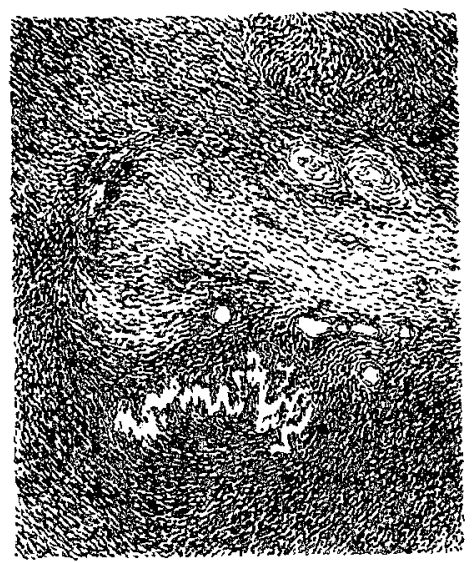

Figur 2.

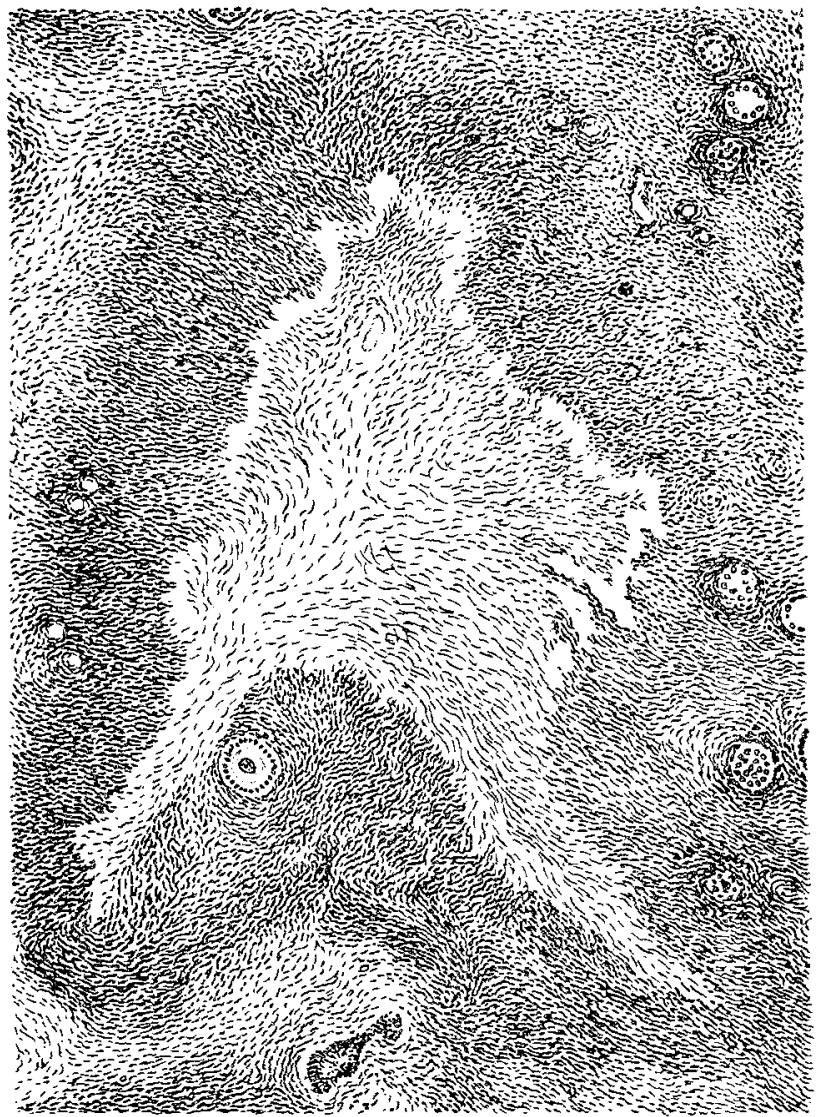


Aus grösseren, nicht geplatżten Follikeln oder aus kleineren, Corpora lutea (menstruationis) können sich die gleichen Gebilde entwickeln, je nachdem mehr das in den Hohlraum wuchernde Bindegewebe oder die hyalin entartete Thecaschicht überwiegt, die ja auch bei atresirenden Follikel, wie Seitz und Cohn gezeigt haben, recht grosse Dimensionen annehmen kann. Im ersten Fall kommt es zu dem Corpus fibrosum simplex, mit wenig oder gar nicht hyalin entarteter Theca interna, dann gehen die Grenzen des Bindegewebskernes ganz allmählich in das umgebende Stroma über. Im 2. Falle erhalten wir das Corpus candicans, bei welchem die Theca interna in Gestalt eines mehr oder weniger umfänglichen, gegen die Umgebung scharf abgesetzten hyalinen Bandes sich erhalten hat, mit mehr oder weniger grossem bindegewebigen Kern. (Tafel XX, Fig. 4 u. 5 u. 6.) Zwischen diesen beiden giebt es noch zahlreiche Uebergänge, je nach dem Grade der hyalinen Umwandlung oder der Bindegewebswucherung und nach dem Alter des Vorgangs. Auf Tafel XX, Fig. 3 finden sich auf einem Gesichtsfeld die verschiedenartigsten Kombinationen dieser Verhältnisse.

Endlich finden wir noch als Endergebnisse der Rückbildung sehr grosser Corpora lutea, wie sie sich zumeist bei Schwangerschaft bilden, das Corpus fibrosum hypertrophicum, wenn der bindegewebige Kern überwiegt, oder, was wohl der gewöhnliche Vorgang ist, das Corpus albicans mit scharfer Abgrenzung gegen die Umgebung und dem charakteristischen sehnigen Glanze.

Ich glaube aus diesen allerdings nur kurz gefassten Schilderungen meines Materiales das eine schliessen zu dürfen, dass alle die mannigfaltigen Formen der Rückbildungsproducte atresirender oder geplatzter Follikel nicht auf die verschiedenartige Genese der bei der Um- und Rüekbildung betheiligten Luteinzellenformationen zurückgeführt zu. werden brauchen, etwa in dem Sinne, dass nur epithelial gebildete Luteinzellen zur Bildung von hyalinen Körpern führen, und die bindegewebig entstandenen nur zur Bildung der Corpora fibrosa, sondern ich glaube, dass die versehiedenen Bildungen nur davon abhängen, ob überhaupt eine kräftige Luteinzellschicht gebildet wird: Eine Unterscheidung zwischen zwei genetisch verschiedenen Luteinzellarten kann ich auf Grund meiner Präparate nicht als bewiesen ansehen, da ich eben gezeigt habe, dass die beiden Zellarten bereits an einem noch epithelbekleideten frisehgeplatzten Follikel nachweisbar sind, und alle Uebergänge ineinander aufweisen. 
544 Hegar, Studien zur Histogenese des Corpus luteum u.s.w.

Das Studium der verschiedenen Rückbildungsvorgänge zeigt aber, dass die Bildung hyaliner Massen stets an die Bildung der Luteinzellschichten auch bei einfach atresirenden Follikeln gebunden ist, die Bildung des Corpus fibrosum aber auf Wucherung des gewöhnlichen Ovarialgewebes beruht. Man kann also den Satz prägen :

Alle corpora candicantia nehmen ihren Ursprung aus der Theca interna, g]eichgültig, ob es sich um die Producte geplatzter oder atresirender Follikel handelt; alle Corpora fibrosa entstammen der Theca externa, und die so häufigen Mischformen entstehen aus der wechselnden Betheiligung beider Elemente. Eine Betheiligung des Epithels an der Bildung der Corpora candicantia ist bis jetzt für den Menschen nicht einwandsfrei bewiesen. Ob dieselbe vorkommt, müssen noch weitere Untersuchungen an frühesten Stadien der Corpus luteum-Bildung zeigen.

Fasse ich die nur kurz gesehilderten histologischen Untersuchungen an den verschiedenen Stadien der Corpus luteum-Bildung zusammen, so möchte ich Folgendes hervorheben:

1. Die von Rabl, später von Seitz, Sohma und Cohn beschriebenen Schichtungen des Luteinzellgewebes bönnen bereits in ihren ersten Anfängen an dem noch epithelhaltigen, frischgeplatzten Follikel nachgewiesen werden. Zwischen beiden Zellschichten bestehen zu allen Zeiten der Entwickelung des gelben Körpers fliessende Uebergänge. Die äussere Zellschicht ist stets nur fleckförmig um die eintretenden Gefässe angeordnet und geht bei weiterer Entwickelung des Corpus luteum vollständig in der inneren Zellschicht auf.

2. Diese nahen Beziehungen beider Zellschichten, sowie der Umstand, dass die innere Luteinzellschicht in allen Entwickelungsstadion des Corpus luteum von einer feinen inneren Grenzbaut bekleidet ist, lassen eine gemeinsame Entstehung der Luteinzellschichten wahrscheinlich erscheinen und zwar aus der Theca interna. Die von Cohn aus der Zweitheilung der Luteinzellschicht gezogenen weitgehenden Schlussfolgerungen über die epitheliale Abkunft der inneren Schicht sind nicht haltbar, und die für die Thiere sicher gestellte epitheliale Abkunft bedarf für den Menschen erst noch des Beweises. 
3. Die mannigfachen Formen der Rückbildungsproducte geplatzter und nicht geplatzter Follikel lassen sich, wie bereits Böshagen gezeigt, aus der verschiedenartigen Betheiligung der Theca interna und der Theca externa einheitlich erklären.

\section{Erklärungen der Abbildungen auf Tafel XX.}

Figur 1. Fall 1. Eben geplatzter Follikel. a) Follikelepithel. b) Ausgebildete Luteinschicht, mit grossen sog. Granulosa-Luteinzellen. c) Fleckweise vertheilte äussere Luteinschicht, sog. Thecaluteinzellen.

Figur 2. Corpus luteum graviditatis (aus Sohma, dieses Archiv, Bd. 84) zeigt drei verschiedene Uebergangsformen von Luteinzellen.

Figur 3. a) Corpus fibrosum mit stark entwickeltem hyalinen Mantel. b) Corpus candicans mit schwachem bindegewebigen Kern.

Figur 4 . Corpus candicans mit hyaliner Krause und bindegewebigem Kern.

Figur 5. Corpora fibrosa mit schwach and ungleichmässig entwickeltem byalinen Band auf verschiedenen Durchschnitten.

Figur 6. Corpus albicans mit allerseits scharfer Abgrenzung gegen die Umgebung. 